\title{
Absorbed Dose to Water Rate in a Cyberknife VSI System Reference Field Using Ionization Chambers and Gafchromic Films
}

\author{
Guerda Massillon-JL1, Nestor Aragón-Martínez¹, Arnulfo Gómez-Muñoz², \\ Abel Hernández-Guzmán' \\ ${ }^{1}$ Instituto de Física, Universidad Nacional Autónoma de México, Ciudad de México, México \\ ${ }^{2}$ Hospital de Oncología, Centro Médico Nacional Siglo XXI, Ciudad de México, México \\ Email: massillon@fisica.unam.mx
}

How to cite this paper: Massillon-JL, G., Aragón-Martínez, N., Gómez-Muñoz, A. and Hernández-Guzmán, A. (2017) Absorbed Dose to Water Rate in a Cyberknife VSI System Reference Field Using Ionization Chambers and Gafchromic Films. International Journal of Medical Physics, Clinical Engineering and Radiation Oncology, 6, 80-92.

https://doi.org/10.4236/ijmpcero.2017.61008

Received: December 16, 2016

Accepted: February 21, 2017

Published: February 24, 2017

Copyright (C) 2017 by authors and Scientific Research Publishing Inc. This work is licensed under the Creative Commons Attribution International License (CC BY 4.0).

http://creativecommons.org/licenses/by/4.0/ c) (i) Open Access

\begin{abstract}
This work investigated the absorbed dose to water rate under reference conditions in a Cyberknife VSI system using radiochromic films EBT3 and MD-V3 and three ionization chambers: an Exradin A12 and two FC65P Welhöfer Scanditronix with different serial numbers. The correction factor, $k_{Q_{\text {msr }}, Q}^{f_{\text {mar }}, f_{\text {ref }}}$, was studied using a Varian iX linac and the Cyberknife system. The measurements in the Varian iX were performed in a $10 \times 10 \mathrm{~cm}^{2}$ field, $10 \mathrm{~cm}$ depth in liquid water at $90 \mathrm{~cm}$ and $70 \mathrm{~cm} \mathrm{SSD}$ and in a $5.4 \times 5.4 \mathrm{~cm}^{2}$ field, $10 \mathrm{~cm}$ depth at $70 \mathrm{~cm}$ SSD to simulate the Cyberknife conditions. In the Cyberknife system, measurements were performed using ionization chambers and both film types at $70 \mathrm{~cm} \mathrm{SSD}$ and $10 \mathrm{~cm}$ depth in its $6 \mathrm{~cm}$ diameter reference field. The results indicate that $k_{Q_{m s} r}^{f_{m s r},}, f_{\text {ref }}$ is independent of the dosimeters and the evaluation methods. Maximum differences of $0.22 \%-0.55 \%$ (combined uncertainties of $1.22 \%-1.98 \%, k=1)$ are obtained on $k_{Q_{m s s}, Q}^{f_{\text {mss }}, f_{\text {ref }}}$ using Varian iX, whereas discrepancies of $2.08 \%-2.09 \%$ (combined uncertainties of $1.87 \%$ $2.13 \%, k=1$ ) are observed using the Cyberknife system. Given the agreement between detectors and the combined standard uncertainties, the data from Varian iX could be considered the most accurate and consequently a weighted average factor of $0.902 \pm 0.006$ could be used for the Cyberknife VSI system reference field. Within measurement uncertainties, the absorbed dose rate measured in the Cyberknife VSI system reference field was found to be independent of the dosimeters used. These results suggest that the absorbed dose measured at a point within a given field size should be the same, regardless the dosimeter used, if their dosimetric characteristics are well known. This highlighted the importance of performing dosimetry by controlling all parameters that could affect the dosimeter response. One can conclude that radiochromic
\end{abstract}


film dosimetry can be considered as an appropriate alternative for measuring absorbed dose to water rate.

\section{Keywords}

Small Field Dosimetry, Reference Field, Cyberknife, Gafchromic Films, Ionization Chambers, Dose Rate, EBT3, MD-V3, 6 MV X-Rays

\section{Introduction}

The therapeutical use of ionizing radiation requires an accurate and precise knowledge of the spatial absorbed dose distribution delivered to the patient for achieving high tumour control rates and at the same time, protecting any adjacent healthy tissue. In particular, the use of small radiation fields $(60 \mathrm{~mm}$ down to 5 or $4 \mathrm{~mm}$ diameter) should impart dose to the target volume in a sure manner. The physical concept of small radiation fields in radiotherapy is strongly correlated to the high-ionization density problem caused by the variation of the electron fluence spectra in the lateral direction and the small range of the electrons generated in the field [1]. Consequently, the determination of the absorbed dose in these fields remains a challenging task compared to the fields used in conventional radiotherapy (square fields from $4 \mathrm{~cm}$ to $40 \mathrm{~cm}$ ). Since the electron fluence spectra change in quality and in quantity when interacting with the medium [2], an ideal dosimeter to measure the absorbed dose in these fields should be small, with high-spatial resolution, water tissue-equivalent, dose-rate and energy independent. Unfortunately, such a dosimeter doesn't exit and the best approach should be a precise knowledge of the dosimetric characteristics of the dosimeters commercially available in order to evaluate their limitations before their use in small radiotherapy fields.

Conventionally, the procedures for reference fields described by the present dosimetry protocols such as IAEA TRS-398 [3] and AAPM TG-51 [4] to measure the absorbed dose to water rate are based on a square field of $10 \mathrm{~cm} \times 10 \mathrm{~cm}$. However, systems like Gammaknife or Cyberknife do not have the possibility to generate such a radiation field size. The main problem to calibrate a Cyberknife unit, where the machine reference field is a circular field of $6 \mathrm{~cm}$ diameter, is the fact that the ionization chamber used is generally calibrated in a $10 \mathrm{~cm} \times 10 \mathrm{~cm}$ square field. So, from a standpoint of radiation physics and dosimetry, the problem is not only related to the size but also to the field geometry. Based on this fact, a new formalism for small and non-standard reference fields has been proposed [5] by introducing the concept of intermediary field as an extension of the existent protocols. In this new formalism, a factor, $k_{Q_{m s}, Q}^{f_{m s}, f_{\text {ref }}}$ that corrects for differences in the ionization chamber response at the intermediary field, $f_{m s r}$ with respect to that of the conventional reference field, $f_{\text {rei }}$ has been added according to the following relation [5]:

$$
D_{w, Q_{m s r}}^{f_{m s r}}=M_{Q_{m s r}}^{f_{m s r}} N_{D, w, Q_{0}} k_{Q, Q_{0}} k_{Q_{m s s}, Q}^{f_{m s r}, f_{r e f}},
$$


with

$$
k_{Q_{m s}, Q}^{f_{m s r}, f_{\text {ref }}}=\frac{D_{w, Q_{m s r}}^{f_{m s r}} / M_{Q_{m s r}}^{f_{m s r}}}{D_{w, Q}^{f_{r e f}} / M_{Q}^{f_{\text {ref }}}},
$$

where $M_{Q_{m s r}}^{f_{m s r}}$ is the corrected reading of the dosimeter in the field $f_{m s i} ; N_{D, w, Q_{0}}$ is the calibration coefficient in terms of absorbed dose to water, $w$, for an ionization chamber calibrated in a $10 \mathrm{~cm} \times 10 \mathrm{~cm}$ reference beam quality $Q_{0}$, and $k_{Q, Q_{0}}$ is the beam-quality correction factor, which corrects for the differences between the reference beam quality $Q_{o}$ at the standard laboratory and the beam quality $Q$ of the conventional reference field $f_{\text {ref }}$

According to this proposal, besides the field size, this new factor also accounts for the differences on geometry, phantom material and beam quality. In addition, it has been suggested to obtain this factor preferably by a direct calibration of the ionization chamber in the two fields against a primary standard or against another dosimeter calibrated in terms of absorbed dose to water traceable to a primary standard without substantial beam quality dependence [5]. Since 2008, after the new formalism proposal was made public, most of the studies have concentrated in relative dosimetry and very few have determined experimentally the absorbed dose to water rate in $m s r$ fields [6] or reported the required correction factor, $k_{Q_{m s s},}^{f_{\text {ms }}, f_{\text {ref }}}$, for reference dosimetry [7] [8]. Massillon-JL and collaborators have proposed the use of Gafchromic films to measure the absorbed dose to water rate in $m s r$ fields by establishing a strict and rigorous dosimetric protocol for film dosimetry [6]. Francescon and collaborators [7] have calculated the product $k_{Q, Q_{0}} k_{Q_{m s}, Q}^{f_{\text {mss }}, f_{\text {ref }}}$ for a $6 \mathrm{MV}$ x-ray Cyberknife $m s r$ field using five ionization chambers through Monte Carlo simulation finding values between 0.999 and 1.006 depending on the chamber type. In their study, the factor was obtained as a ratio of the absorbed dose in water and the absorbed dose in the air volume of the chamber at $75 \mathrm{~cm} \mathrm{SSD}$ and $5 \mathrm{~cm}$ depth in a $6 \mathrm{~cm}$ diameter $m s r$ field relative to that for the ${ }^{60} \mathrm{Co}$ gamma at $100 \mathrm{~cm} \mathrm{SSD}$ and $5 \mathrm{~cm}$ depth in a 10 $\mathrm{cm} \times 10$ square field [7]. Independently, Pantelis and collaborators [8] have reported a correction factor $k_{Q_{m s s}, Q}^{f_{\text {msr }}, f_{\text {ref }}}=0.999 \pm 0.016$ for a Farmer type chamber obtained as a ratio of the absorbed dose measured with the Farmer chamber and alanine dosimeter in a $6 \mathrm{~cm}$ Cyberknife $m r s$ field at $75 \mathrm{~cm} \mathrm{SSD}$ and $5 \mathrm{~cm}$ water depth.

This work has investigated, through a $6 \mathrm{MV} \mathrm{x}$-ray Varian iX linear accelerator, the $k_{Q_{m s}, Q}^{f_{m s}, f_{\text {ref }}}$ factor for a $6 \mathrm{MV}$ x-ray Cyberknife unit equivalent $m s r$ field $(5.4 \mathrm{~cm} \times 5.4 \mathrm{~cm})$ using three ionization chambers and Gafchromic films. The absorbed dose to water rate was determined through Equation (1) using ionization chambers in the Cyberknife unit reference field $(6 \mathrm{~cm}$ diameter) and compared with that measured directly with calibrated Gafchromic films.

\section{Material and Methods}

\subsection{Preparation and Readout of the Gafchromic Films}

Gafchromic films type EBT3 (lote \#07221301) and MD-V3 (lote \#A03051201) 
were cut into pieces of different size ranging from $1.2 \mathrm{~cm} \times 1.2 \mathrm{~cm}$ up to $7 \mathrm{~cm} \times$ $7 \mathrm{~cm}$, depending on the need. This process was done at least 24 hours prior irradiation. Twenty-four hours posterior to irradiation, the film was read in transmission mode using an Epson Expression 11000XL document scanner at $300 \mathrm{dpi}$ and 48 bit-RGB colour depths following the protocol reported previously [9] [10]. The analysis of the film response was done using Image J software [11] according to the method described and reported elsewhere [10].

\subsection{Measurement in a 6 MV X-Ray Varian iX Linear Accelerator}

\subsubsection{Calibration of a $10 \mathrm{~cm} \times \mathbf{1 0} \mathrm{cm}$ Reference Field}

The absorbed dose to water rate was measured according to the IAEA TRS-398 protocol [3] in a $10 \mathrm{~cm} \times 10 \mathrm{~cm}$ field at $90 \mathrm{~cm}$ source to surface distance (SSD) and $10 \mathrm{~cm}$ depth in liquid water using a PTW-MP3 phantom. Three ionization chambers (IC) calibrated in different laboratories were used: a) An Exradin A12 associated with a Standard Imaging Supermax 90018 electrometer calibrated at the National Institute of Standards and Technology, NIST, USA, herein IC-A12; b) a FC65P Welhöfer Scanditronix (SN2258) coupled with a Dose One (SN17237) electrometer calibrated at IBA, Germany, called IC-2258 and; c) a FC65P Welhöfer Scanditronix (SN580) connected with a Dose One (SN7969) electrometer calibrated at ININ, Mexico, defined as IC-580. Being calibrated at a primary standard laboratory, the IC-A12 was considered as our reference dosimetry system. For a better accuracy on the measurement, the exposure of the ICs was performed consecutively and the ambient temperature and pressure were monitored simultaneously using a calibrated and high precision digital thermometer Fluke 1523 and a Druck DPI 12 barometer, respectively. Every single charge collected was corrected for pressure and temperature differences between the calibration laboratory and the user conditions.

\subsubsection{Calibration of the Gafchromic Films}

Three $1.2 \mathrm{~cm} \times 1.2 \mathrm{~cm}$ pieces from each Gafchromic film type EBT3 and MD-V3 were irradiated simultaneously in liquid water at each absorbed dose, at values from 0 to $60 \mathrm{~Gy}$, using the absorbed dose rate obtained with the IC-A12 dosimetry system. All film pieces were vacuum-sealed and statistic agreement within $0.65 \%$ (less than measurement uncertainties) has been reported between the absorbed dose to water inside the waterproof package and that measured directly in the water phantom [6]. The irradiation of the films in liquid water (Figure 1) was performed according to our protocol [6]. The measurements were carried out under the same conditions of the reference calibration; i.e. 10 $\mathrm{cm} \times 10 \mathrm{~cm}$ field at $90 \mathrm{~cm} \mathrm{SSD}$ and $10 \mathrm{~cm}$ depth in liquid water using a PTWMP3 phantom.

\subsection{Determination of the $k_{Q_{m s r}, Q}^{f_{m s r}, f_{\text {ref }}}$ Factor}

To determine the $k_{Q_{m s r}, Q}^{f_{m s}, f_{\text {ref }}}$ factor, two experimental methods were considered: 


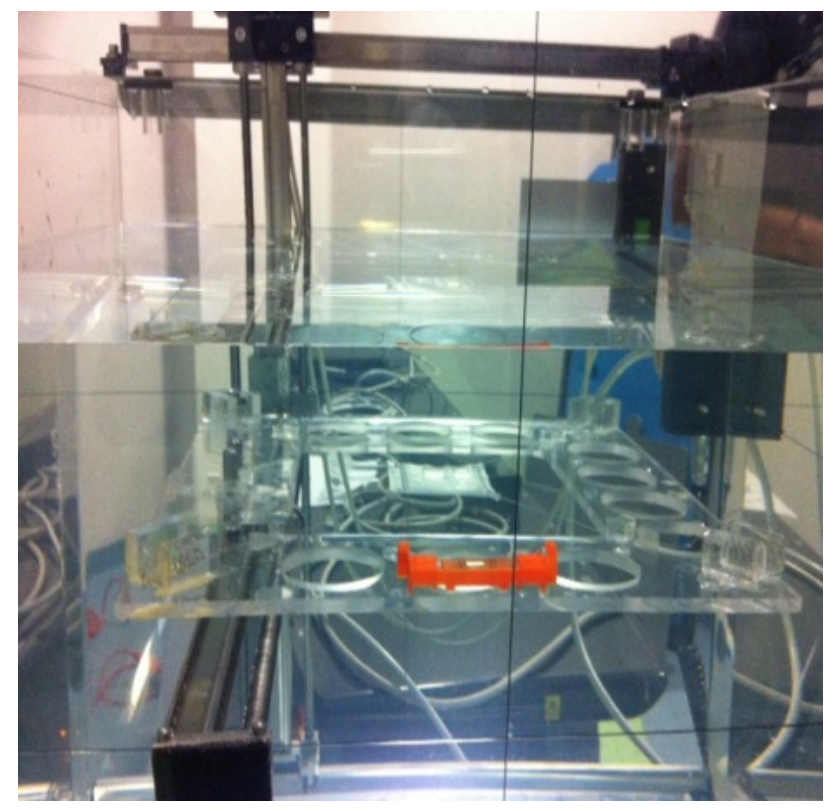

Figure 1. Irradiation setup of the radiochromic films in liquid water. The films were always oriented perpendicular to the beam.

\subsubsection{Method I: Measurement in a Square Equivalent Field of a Varian iX Linac}

Compared with conventional linear accelerator (linac) which has flattening filter and allows measurement at a source to detector distance (SDD) of $100 \mathrm{~cm}$, the Cyberknife unit has no beam-flattening filter and is limited to SDD $=80 \mathrm{~cm}$ [12]. This means that the contribution of low-energy scattering radiation caused by the collimators and/or multi-leaf system to the dosimeter response when measurements are performed very close to a conventional linac head should be equivalent to that from a Cyberknife unit, i.e. the photon energy spectra should be similar. If this assumption is correct, the $k_{Q_{\text {msr }}, Q}^{f_{\text {ms }}, f_{\text {ref }}}$ factor measured in a square equivalent field from the linac must be similar to that obtained in the 6 $\mathrm{cm}$ diameter reference field from the Cyberknife unit under the same conditions. Based on this consideration, the Cyberknife geometry conditions were mimicked using a $6 \mathrm{MV}$-ray beam produced in a Varian iX linac by measuring the absorbed dose in a $10 \mathrm{~cm} \times 10 \mathrm{~cm}$ and $5.4 \mathrm{~cm} \times 5.4 \mathrm{~cm}$ square fields at $\mathrm{SDD}=80$ $\mathrm{cm}$ and $10 \mathrm{~cm}$ depth in liquid water using a PTW-MP3 phantom. The $5.4 \mathrm{~cm} \times$ $5.4 \mathrm{~cm}$ field is the square equivalent to $6 \mathrm{~cm}$ diameter reference field of the Cyberknife unit calculated according to the following relation [13]:

$$
l=d(0.891+0.00046 d),
$$

where $l$ is the length of the equivalent square field and $d$, the diameter of the circular field.

The absorbed doses were measured in both field sizes with the ICs mentioned above and the Gafchromic EBT3 and MD-V3 films. For the ICs exposure, the same procedure from section 2.2.1 was followed, whereas the EBT3 film was exposed to 704 Monitor units (MU), and the MD-V3 to $1409 \mathrm{MU}$. The selection of these MU was based on the analysis of the calibration curve for each film where 
the combined standard uncertainty in the absorbed dose was less than or equal to $2 \%$ for a coverage factor $k=1$. After irradiation of the films, the same procedure used for the calibration curve was followed during the reading process and the obtained netOD was converted into absorbed dose using the calibration reference curve. Thus, the factor that corrects for differences in the ionization chamber response at the intermediary field, $f_{m s s}$, with respect to that of the conventional reference field, $f_{\text {ref }}$ has been evaluated as follow:

$$
k_{Q_{m s}, Q}^{f_{m s}, f_{\text {ref }}}=\frac{D_{5.4 \mathrm{~cm}} / R_{5.4 \mathrm{~cm}}}{D_{10 \mathrm{~cm}} / R_{10 \mathrm{~cm}}}
$$

where $D_{5.4 \mathrm{~cm}}$ and $R_{5.4 \mathrm{~cm}}$ are the measured absorbed dose and the corresponding response of a given dosimeter in the equivalent radiation field, respectively. $D_{10 \mathrm{~cm}}$ and $R_{10 \mathrm{~cm}}$ represent the measured absorbed dose and the response of the same dosimeter in the reference field of $10 \mathrm{~cm} \times 10 \mathrm{~cm}$. This factor (Equation (4)), in principle, could be evaluated with any well-calibrated dosimeter type.

\subsubsection{Method II: Measurement in the $6 \mathrm{~cm}$ Diameter Reference Field of the Cyberknife System}

This method is similar to the one used by Pantelis and collaborators [8] and consisted on determining the $k_{Q_{m s r}, Q}^{f_{\text {msr }} f_{\text {ref }}}$ factor as a ratio of the absorbed dose measured with the ionization chambers following the conventional IAEA-398 code of practice and that measured with calibrated Gafchromic film types EBT3 and MD-V3 under the same conditions. For that, the absorbed dose was measured with the ICs IC-A12 and IC-580 in the $6 \mathrm{~cm}$ diameter reference field in the Cyberknife unit at $70 \mathrm{~cm} \mathrm{SSD}$ and $10 \mathrm{~cm}$ water depth using an IBA blue phantom. Similarly, pieces of EBT3 and MD-V3 films were exposed directly in liquid water to $1502 \mathrm{MU}(\sim 10 \mathrm{~Gy})$ and $3003 \mathrm{MU}$ ( 20 Gy), respectively. Once again, the same process mentioned above was followed to determine the absorbed dose delivered to the films. Considering that the absorbed dose at a certain point within a radiation field should be independent of the dosimeter used, the correction factor was evaluated from Equation (1) as:

$$
D_{w, Q_{m s r}}^{f_{m s r}}=D_{\text {Films }} \Rightarrow k_{Q_{m s r}, Q}^{f_{m s}, f_{r e f}}=\frac{D_{\text {Films }}}{M_{Q_{m s r}}^{f_{m s r}} N_{D, w, Q_{0}} k_{Q, Q_{0}}}
$$

where, $M_{Q_{m s r}}^{f_{m s r}}, N_{D, w, Q_{0}}$ and $k_{Q, Q_{0}}$ have the same meaning as in Equation (1), while $D_{\text {Films }}$ is the absorbed dose measured with the Gafchromic films in the same beam. In this work the beam quality $Q$ and $Q_{0}$ represent $6 \mathrm{MV}$ x-rays and ${ }^{60}$ Co gamma, respectively.

To minimize measurement uncertainties, the beam-quality correction factor in the x-ray beams was determined by fitting the $k_{Q, Q_{0}}$ values as a function of the tissue-phantom ratio $\left(T P R_{20,10}\right)$ data provided by the IAEA-398 code of practice [3] for each ionization chamber using the following relation:

$$
k_{Q, Q_{0}}=a+b T P R_{20,10}^{C}
$$

where $a, b$ and $c$ are fit parameters, whereas $T P R_{20,10}$ is the corresponding measurement in the radiation beam in question. 


\subsection{Determination of the Absorbed Dose in the $6 \mathrm{~cm}$ Diameter Reference Field of the Cyberknife System}

The measurements were performed in the $6 \mathrm{~cm}$ diameter field at $70 \mathrm{~cm} \mathrm{SSD}$ and $10 \mathrm{~cm}$ liquid water depth using the dosimetric systems IC-A12 and IC-580 following the IAEA TRS-398 protocol [3]. Thereafter, the absorbed dose in the reference field was obtained using the measured correction factor, $k_{Q_{m s}, Q}^{f_{m s}, f_{\text {ref }}}$, according to Equation (1) [5]. Due to a technical problem, the dosimetric system IC-2258 was not used in this case. For comparison, the dose rate was measured directly in liquid water with radiochromic films EBT3 and MD-V3 under the same conditions as the ionization chambers [6], that is, $70 \mathrm{~cm} \mathrm{SSD,} 10 \mathrm{~cm}$ water depth and $6 \mathrm{~cm}$ diameter field. The films were exposed to the same MU as mentioned in section 2.3.2 above.

\section{Results}

The absorbed dose rates measured in the $10 \times 10 \mathrm{~cm}$ reference field from the 6 MV x-ray Varian iX linac are shown in Table 1 for the ionization chambers. As observed, within measurement uncertainties, there exists good agreement between our dosimetric system and the IC-2258, however a slight difference is observed on the absorbed dose rate measured with the same ionization chamber model (IC-580 vs. IC-2258) calibrated in different laboratories. Table 2 displays the absorbed dose rate measured at $70 \mathrm{~cm} \mathrm{SSD} \mathrm{for} \mathrm{the} 10 \mathrm{~cm} \times 10 \mathrm{~cm}$ and $5.4 \mathrm{~cm}$ $\times 5.4 \mathrm{~cm}$ square field. Note an increase in the dose rate measured with IC-580 and IC-2258 relative to IC-A12, independent of the field size. This is associated to the low-energy scattered radiation from the collimators and/or the head of the linac.

The calibration curves for both films are displayed in Figure 2(a) and Figure 2(b) for MD-V3 and EBT3, respectively. As seen within the absorbed dose interval studied, the response of the MD-V3 film is described by a second-order

Table 1. Absorbed dose to water rate measured in the Varian iX linac under reference conditions: SSD $90 \mathrm{~cm}$ and $10 \mathrm{~cm}$ depth for the $10 \mathrm{~cm} \times 10 \mathrm{~cm}$ field. The combined standards uncertainties correspond to a coverage factor $k=1$ [14].

\begin{tabular}{ccccc}
\hline Ionization Chamber & TPR $_{20,10}$ & $k_{Q, Q_{0}}$ & Dose Rate [cGy/MU] & Other/IC-A12 \\
\hline IC-A12 & 0.6793 & $0.9947 \pm 0.0002$ & $0.785 \pm 0.004$ & 1.000 \\
IC-2258 & 0.6658 & $0.9934 \pm 0.0004$ & $0.787 \pm 0.010$ & 1.003 \\
IC-580 & 0.6632 & $0.9937 \pm 0.0004$ & $0.804 \pm 0.010$ & 1.024 \\
\hline
\end{tabular}

Table 2. Absorbed dose rate to water measured in Varian ${ }^{\oplus}$ iX simulating the Cyberknife ${ }^{\circledast}$ Unit conditions: SSD $70 \mathrm{~cm}$ and $10 \mathrm{~cm}$ depth. The combined standards uncertainties correspond to a coverage factor $k=1$ [14].

\begin{tabular}{ccccccc}
\hline & \multicolumn{3}{c}{$10 \mathrm{~cm} \times 10 \mathrm{~cm}$} & \multicolumn{2}{c}{$5.4 \mathrm{~cm} \times 5.4 \mathrm{~cm}$} \\
\hline Ionization chamber & $\mathrm{TPR}_{20,10}$ & $k_{Q, Q_{0}}$ & Dose Rate [cGy/MU] & $\mathrm{TPR}_{20,10}$ & $k_{Q, Q_{0}}$ & Dose Rate [cGy/MU] \\
IC-A12 & 0.6200 & $0.9987 \pm 0.0001$ & $1.262 \pm 0.007$ & 0.5923 & $0.9997 \pm 0.0001$ & $1.023 \pm 0.005$ \\
IC-2258 & 0.6191 & $0.9975 \pm 0.0002$ & $1.278 \pm 0.014$ & 0.5885 & $0.9991 \pm 0.0001$ & $1.037 \pm 0.011$ \\
IC-580 & 0.6203 & $0.9974 \pm 0.0001$ & $1.311 \pm 0.013$ & 0.5897 & $0.9991 \pm 0.0001$ & $1.059 \pm 0.011$ \\
\hline
\end{tabular}




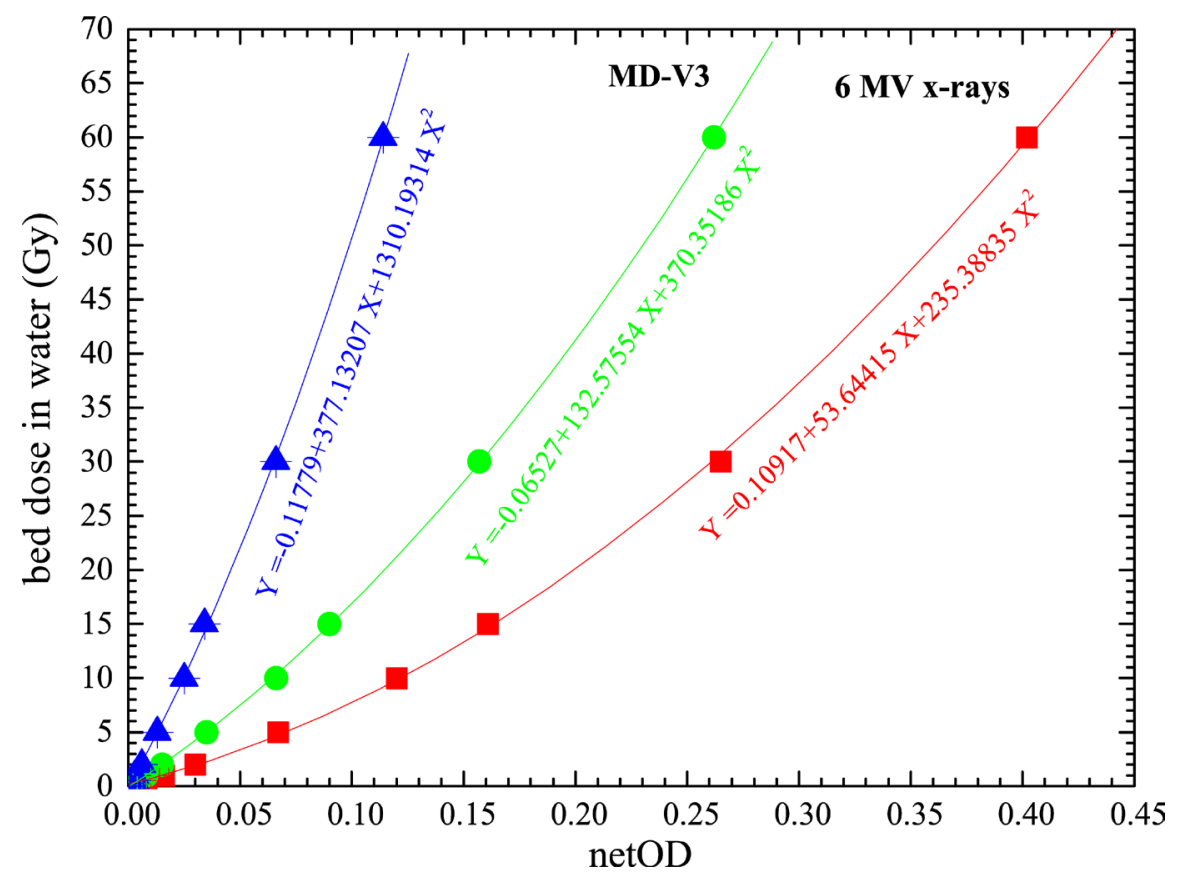

(a)

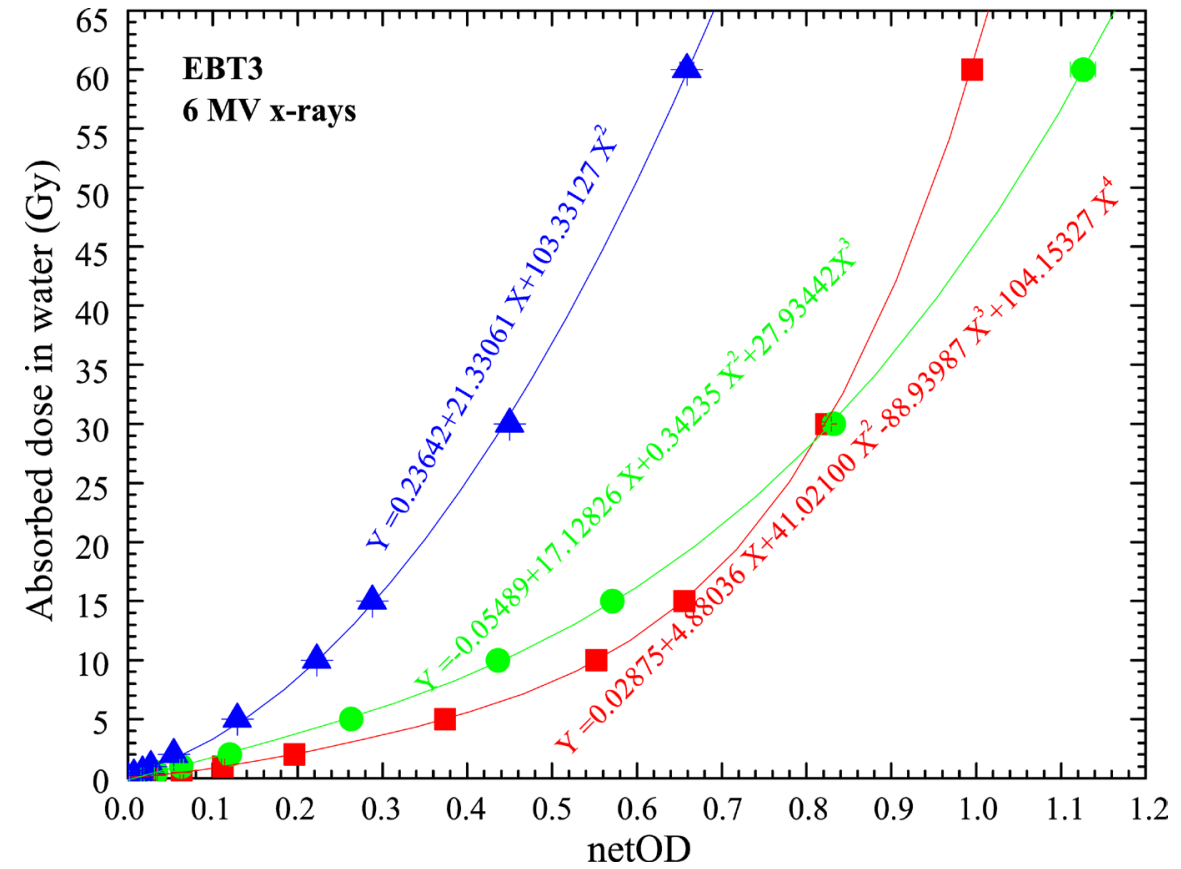

(b)

Figure 2. (a) Calibration curve of the MD-V3 film exposed in liquid water to $6 \mathrm{MV}$ x-rays. (b) Calibration curve of the EBT3 film exposed in liquid water to $6 \mathrm{MV}$ x-rays.

polynomial function independent of the colour channel, while the EBT3 film response in the blue, green and red channel was described by a fourth, third and second-order polynomial function, respectively, which is related to the difference on the dynamic range of both films.

The correction factors evaluated with Equations (4) and (5) using several dosimeters are shown in Table 3. As it can be noted, within measurement uncer- 
tainties, this factor is independent of the dosimeter and the evaluation method. The absorbed dose rate measured in the Cyberknife reference field is shown in Table 4. No statistically significant difference is observed between the ionizations chambers and the Gafchromic films. Also shown in Table 4 are the $T P R_{20,10}$ and the $k_{Q, Q_{0}}$ measured in this work compared with data published in the literature. A good agreement is observed.

\section{Discussion}

In this work, the absorbed dose to water rate in a $6 \mathrm{~cm}$ diameter reference field of a Cyberknife VSI system has been investigated using ionization chambers and Gafchromic films. The absorbed dose rate for the $10 \times 10 \mathrm{~cm}^{2}$ reference field at $90 \mathrm{~cm}$ SSD in a $6 \mathrm{MV}$-rays Varian iX linac using 3 ionization chambers has been measured, finding differences of $0.25 \%$ (combined standard uncertainties $0.51 \%-1.27 \%, k=1$ ) and $2.42 \%$ (combined standard uncertainties $0.51 \%$ $1.24 \%, k=1)$ between IC-A12 and IC-2258, and IC-580 and IC-A12, respectively. Curiously, discrepancy of $2.11 \%$ (combined standard uncertainties of $1.24 \%-1.27 \%, k=1$ ) is observed between the chambers from the same model and different serial numbers. Considering that all measurements were performed simultaneously under similar conditions, such a disagreement could only be attributed to the absorbed dose calibration coefficient, $N_{D, w^{*}}$ As observed in Table 2 and independent of the field size, ionization chambers IC-2258 and

Table 3. $k_{Q_{m s}, Q}^{f_{m s}, f_{\text {ref }}}$ measured in a linac Varian ${ }^{\circledast} \mathrm{iX}$ (Equation (4)) and in the Cyberknife unit (Equation (5)). The uncertainties correspond to a coverage factor $k=1$ [14].

\begin{tabular}{cccc}
\hline & Method I & \multicolumn{2}{c}{ Method II } \\
\cline { 3 - 4 } Dosimeter & & MD-V3 & EBT3 \\
& $0.901 \pm 0.011$ & $0.913 \pm 0.018$ & $0.908 \pm 0.017$ \\
IC-A12 & $0.903 \pm 0.014$ & & \\
IC-2258 & $0.899 \pm 0.013$ & $0.894 \pm 0.019$ & \\
IC-580 & $0.905 \pm 0.016$ & & \\
EBT3 & $0.906 \pm 0.018$ & & \\
MD-V3 & $0.902 \pm 0.006$ & $0.904 \pm 0.013$ & $0.899 \pm 0.012$ \\
\hline
\end{tabular}

Table 4. Absorbed dose to water rate measured in Cyberknife ${ }^{\circledast}$ Unit with ionization chambers and Gafchromic films. The uncertainties correspond to a coverage factor $k=1$ [14].

\begin{tabular}{cccccc}
\hline \multirow{2}{*}{ Dosimeter } & \multicolumn{2}{c}{ Kawachi et al. 2008 [15] } & \multicolumn{2}{c}{ This work } & $\begin{array}{c}\text { Dose rate } \\
\text { [cGy/MU] }\end{array}$ \\
\cline { 2 - 5 } & $\mathrm{TPR}_{20,10}$ & $k_{Q, Q_{0}}$ & $\mathrm{TPR}_{20,10}$ & $k_{Q, Q_{0}}$ & $0.666 \pm 0.009$ \\
\hline IC-A12 & 0.64 & 0.995 & 0.6448 & $0.9974 \pm 0.0001$ & $0.0 .9973 \pm 0.0001$ \\
IC-580 & 0.64 & 0.994 & 0.6467 & $0.679 \pm 0.012$ \\
EBT3 & & & & & $0.671 \pm 0.012$ \\
MD-V3 & & & & & $0.675 \pm 0.013$ \\
\hline
\end{tabular}


IC-580 depict an over-response relative to IC-A12 as the SSD decreases. That is, the absorbed dose rates measured at $70 \mathrm{~cm}$ SSD with IC-2258 are greater than those measured with the IC-A12 by $1.27 \%$ and $1.37 \%$ for the $10 \times 10 \mathrm{~cm}^{2}$ and 5.4 $\times 5.4 \mathrm{~cm}^{2}$ fields, respectively. The data obtained with IC-580 are greater than those measured with the IC-A12 by $3.88 \%$ and $3.52 \%$ for the $10 \times 10 \mathrm{~cm}^{2}$ and 5.4 $\times 5.4 \mathrm{~cm}^{2}$ fields, respectively. Note that, comparing to the measurement at $90 \mathrm{~cm}$ SSD, the differences between IC-2258 and IC-580 remain statistically unchanged at $70 \mathrm{~cm} \mathrm{SSD}$; i.e. variations of $2.51 \%$ and $2.10 \%$ for the $10 \times 10 \mathrm{~cm}^{2}$ and $5.4 \times$ $5.4 \mathrm{~cm}^{2}$ fields, respectively. Such result supports our previous argument related to the calibration coefficient. The greater response obtained for the IC-2258 and IC-580 chambers with respect to the IC-A12 could be related to the energy dependence of these chambers with low photon energies. That is, at $70 \mathrm{~cm}$ the air volume between the source and the water surface phantom is smaller and the contribution of low-energy radiation is more important due to a lack of low-energy photon fluence attenuation [16]. Comparing the data from Table 1 and Table 2 for the $10 \times 10 \mathrm{~cm}^{2}$ field, $T P R_{20,10}$ decreases as the SSD diminishes with differences of $8.7 \%, 7.0 \%$ and $6.5 \%$ which, are translated into a slight variation on the beam quality correction factor, $k_{Q, Q_{0}}$ of about $0.40 \%, 0.41 \%$ and $0.37 \%$ for the IC-A12, IC-2258 and IC-580 chambers, respectively. This suggests that the difference observed in the chamber response due to the contribution of the low-energy photon beam is not accurately reflected on the $k_{Q, Q_{0}}$ values reported by the IAEA-TRS-398 [3]. Furthermore, the data in Table 2 indicated that the beam quality correction factor, $k_{Q, Q_{0}}$ slightly varies with the field size. That is, between the $10 \times 10 \mathrm{~cm}^{2}$ and the $5.4 \times 5.4 \mathrm{~cm}^{2}$ fields, the variations are $0.10 \%, 0.16 \%$ and $0.17 \%$ for the IC-A12, IC-2258 and IC-580, respectively.

Interestingly, the differences observed between chamber responses on the absorbed dose rate measurements do not affect the correction factor, $k_{Q_{m s},}^{f_{m s}, f_{\text {ref }}}$, shown in Table 3. Such behaviour suggests that the change on the SSD affects considerably more the contribution of low photon energies to the main radiation beam than the change on the field sizes, at least for those considered in this study. Data in Table 3 suggests that within measurement uncertainties, $k_{Q_{m s}, Q}^{f_{\text {ms }}, f_{\text {ref }}}$ is independent of the dosimeters and the evaluation methods, contrasting with the results obtained by Monte Carlo simulation and reported in the literature [7]. According to Table 3, maximum differences of $0.22 \%-0.55 \%$ (combined standard uncertainties of $1.22 \%-1.98 \%, k=1$ ) are obtained with method I, whereas discrepancies of $2.08 \%-2.09 \%$ (combined standard uncertainties of $1.87 \%-2.13 \%, k=1$ ) are observed with method II. Given the level of agreement between detectors and the combined standard uncertainties, the data from method I could be considered the most accurate and consequently a weighted average factor of $0.902 \pm 0.006$ can be considered for the Cyberknife VSI system reference field. Regarding the absorbed dose to water rate displayed in Table 4, within measurement uncertainties no difference is observed between IC-A12 and the films (variations $0.75 \%-1.35 \%$ vs. combined standard uncertainties of $1.35 \%-1.93 \%, k=1)$. However and similarly to the data shown in Table 1 and 
Table 2, the $2 \%$ difference observed between the two ionization chambers was expected due to the argument mentioned above. Note in Table 4 , the $T P R_{20,10}$ measured in the $6 \mathrm{~cm}$ diameter of the Cyberknife VSI system reference field are closer (discrepancies $\sim 4 \%$ ) to those measured for the $10 \times 10 \mathrm{~cm}^{2}$ than the $5.4 \times$ $5.4 \mathrm{~cm}^{2}$ equivalent field (discrepancies $\sim 8 \%$ ), independent of the ionization chamber. However, the influence of this difference on the beam quality factor $k_{Q, Q_{0}}$ is negligible (variations of $\sim 0.23 \%-0.18 \%$ between $5.4 \times 5.4 \mathrm{~cm}^{2}$ and the $6 \mathrm{~cm}$ diameter fields, depending on the chamber), which agree with our previous analysis above.

Comparing our data with those calculated through Monte Carlo simulation and previously reported in the literature [15] displayed in Table 4, good agreement can be observed where variations of $0.74 \%-1.04 \%$ and $0.24 \%-0.33 \%$ are obtained for the $T P R_{20,10}$ and $k_{Q, Q_{0}}$ respectively, depending on the ionization chambers. These small differences could be possibly attributed to the variation of the photon spectra and/or the ionization chamber serial numbers, as in the case of IC-2258 and IC-580 presented in this work.

\section{Conclusion}

The absorbed dose to water rate under reference conditions in a CyberKnife VSI system has been investigated using radiochromic films EBT3 and MD-V3 and three ionization chambers. In particular, the correction factor, $k_{Q_{m s s}, Q}^{f_{m s}, f_{\text {ref }}}$, proposed in the new formalism [5] has been studied through two experimental methods. The results indicate that $k_{Q_{m s}, Q}^{f_{m s r}, f_{\text {ref }}}$ is independent of the dosimeters and the evaluation methods. Besides that and within measurement uncertainties, the absorbed dose rate measured in the CyberKnife VSI system reference field was found to be independent of the dosimeters used. These results suggest that the absorbed dose measured at a point within a given field size should be the same, regardless the dosimeter used (IC or radiochromic films) if their dosimetric characteristics are well known. This highlights the importance of performing dosimetry by controlling all the parameters that could affect the dosimeter response. One can conclude that, under these conditions, radiochromic film dosimetry can be considered as an appropriate alternative for measuring absorbed dose to water rate.

\section{Acknowledgements}

The authors acknowledge Prof. Maria Ester Brandan for revising the manuscript, M. Sc. Eduardo López-Pineda for technical support. This work was partially supported by Royal Society-Newton Advanced Fellowship grant NA150212 and PAPIIT-UNAM grant IN115117.

\section{References}

[1] Cabrera-Santiago, A. and Massillon-JL, G. (2016) Track-Average LET of Secondary Electrons Generated in LiF: Mg, Ti and Liquid Water by $20-300 \mathrm{kV}$ X-Ray, ${ }^{137} \mathrm{Cs}$ and ${ }^{60}$ Co Beams. Physics in Medicine and Biology, 61, 7919-7933. 
https://doi.org/10.1088/0031-9155/61/22/7919

[2] Massillon-JL, G., Minniti, R., Mitch, M.G., Maryanski, M.J. and Soares, C.G. (2009) Use of Gel Dosimetry to Measure the 3D Dose Distribution of a 90Sr/90Y Intravascular Brachytherapy Seed. Physics in Medicine and Biology, 54, 1661-1672. https://doi.org/10.1088/0031-9155/54/6/017

[3] IAEA (2005) Absorbed Dose Determination in Photon and Electron Beams; An International Code of Practice. Technical Report Series, Vol. 277, Viena.

[4] Almond, P.R., Biggs, P.J., Coursey, B.M., Hanson, W.F., Huq, M.S., et al. (1999) AAPM's TG-51 Protocol for Clinical Reference Dosimetry of High-Energy Photon and Electron Beams. Medical Physics, 26, 1847-1870.

https://doi.org/10.1118/1.598691

[5] Alfonso, R., Andreo, P., Capote, R., Huq, M.S., Kilby, W., et al. (2008) A New Formalism for Reference Dosimetry of Small and Nonstandard Fields. Medical Physics, 35, 5179-5186. https://doi.org/10.1118/1.3005481

[6] Massillon-JL, G., Cueva-Procel, D., Diaz-Aguirre, P., Rodriguez-Ponce, M. and Herrera-Martinez, F. (2013) Dosimetry for Small Fields in Stereotactic Radiosurgery Using Gafchromic MD-V2-55 Film, TLD-100 and Alanine Dosimeters. PLoS ONE, 8, e63418. https://doi.org/10.1371/journal.pone.0063418

[7] Francescon, P., Kilby, W., Satariano, N. and Cora, S. (2012) Monte Carlo Simulated Correction Factors for Machine Specific Reference Field Dose Calibration and Output Factor Measurement Using Fixed and Iris Collimators on the Cyber Knife System. Physics in Medicine and Biology, 57, 3741-3758.

https://doi.org/10.1088/0031-9155/57/12/3741

[8] Pantelis, E., Moutsatsos, A., Zourari, K., Kilby, W., Antypas, C., Papagiannis, P., Karaiskos, P., Georgiou, E. and Sakelliou, L. (2010) On the Implementation of a Recently Proposed Dosimetric Formalism to a Robotic Radiosurgery System. Medical Physics, 37, 2369-2379. https://doi.org/10.1118/1.3404289

[9] Massillon-JL, G. and Zúñiga-Meneses, L. (2010) The Response of the New MD-V2-55 Radiochromic Film Exposed to ${ }^{60} \mathrm{Co}$ Gamma Rays. Physics in Medicine and Biology, 55, 5437-5449. https://doi.org/10.1088/0031-9155/55/18/011

[10] Massillon-JL, G., Muñoz-Molina, I.D. and Díaz-Aguirre, P. (2016) Optimum Absorbed Dose versus Energy Response of Gafchromic EBT2 and EBT3 Films Exposed to $20-160 \mathrm{Kv} \mathrm{X}$-Rays and ${ }^{60} \mathrm{Co}$ Gamma. Biomedical Physics \& Engineering Express, 2, Article ID: 045005.

[11] Image J (2016). http://imagej.nih.gov/ij/

[12] Kilby, W., Dooley, J.R., Kuduvalli, G., Sayeh, S. and Maurer, J.R. (2010) The CyberKnife Robotic Radiosurgery System in 2010. Technology in Cancer Research and Treatment, 9, 433-452. https://doi.org/10.1177/153303461000900502

[13] Bewley, D.K., Bradshaw, A.L., Burns, J.E., Cohen, M., Day, M.J., Godden, T.J., Greene, D., Jennings, W.A., Lillicrap, S.C., Smith, C.W. and Williams, P.C. (1996) Central Axis Depth Dose Data for Use in Radiotherapy. The British Journal of Radiology, 104, 114.

[14] ANSI/NCSL Z540-2-1997 1997 U.S. Guide to the Expression of Uncertainty in Measurement. American National Standard for Expressing Uncertainty NCSL, Boulder.

[15] Kawachi, T., Saitoh, H., Inoue, M., Katayose, T., Myojoyama, A. and Hatano, K. (2008) Reference Dosimetry Condition and Beam Quality Correction Factor for CyberKnife Beam. Medical Physics, 35, 4591-4598.

https://doi.org/10.1118/1.2978228 
[16] Aragón-Martínez, N., Gómez-Muñoz, A. and Massillon-JL, G. (2014) Characterization of Radiation Beams Used to Determinate the Correction Factor for a CyberKnife Unit Reference Field Using Ionization Chambers. AIP Conference Series, $1626,55-60$.

Submit or recommend next manuscript to SCIRP and we will provide best service for you:

Accepting pre-submission inquiries through Email, Facebook, LinkedIn, Twitter, etc. A wide selection of journals (inclusive of 9 subjects, more than 200 journals)

Providing 24-hour high-quality service

User-friendly online submission system

Fair and swift peer-review system

Efficient typesetting and proofreading procedure

Display of the result of downloads and visits, as well as the number of cited articles Maximum dissemination of your research work

Submit your manuscript at: http://papersubmission.scirp.org/

Or contact ijmpcero@scirp.org 